\title{
Protein enhanced NIR-IIb emission of indocyanine green for functional bioimaging
}

Mubin $\mathrm{He}^{\mathrm{a}}$, Di Wu ${ }^{\mathrm{b}}$, Yuhuang Zhang ${ }^{\mathrm{a}}$, Xiaoxiao Fan ${ }^{\mathrm{b}}$, Hui Lin ${ }^{\mathrm{b}}$, Jun Qian ${ }^{\mathrm{a}^{*}}$

a. State Key Laboratory of Modern Optical Instrumentations, Centre for Optical and Electromagnetic Research, College of Optical Science and Engineering, International Research Center for Advanced Photonics, Zhejiang University, Hangzhou 310058, China.

b. Department of General Surgery, Sir Run-Run Shaw Hospital, School of Medicine, Zhejiang University, Hangzhou, 310016, China.

*Corresponding author: Jun Qian (qianjun@zju.edu.cn).

\begin{abstract}
Fluorescence imaging performed in the $1500-1700 \mathrm{~nm}$ spectral range (labeled as near-infrared IIb, NIR-IIb) promises high imaging contrast and spatial resolution for its little photon scattering effect and minimum auto-fluorescence. Though inorganic and organic probes have been developed for NIR-IIb bioimaging, most are in preclinical stage, hampering further clinical application. Herein, we showed that indocyanine green (ICG), an US Food and Drug Administration (FDA)-approved agent, exhibited remarkable amount of NIR-IIb emission when dissolved into different protein solutions, including human serum albumin, rat bile, and fetal bovine serum. We performed fluorescence imaging in NIR-IIb window to visualize structures of lymph system, extrahepatic biliary tract and cerebrovascular. Results demonstrated that proteins promoted NIR-IIb emission of ICG in vivo and that NIR-IIb imaging with ICG preserved higher signal-to-background ratio (SBR) and spatial resolution compared with the conventional near-infrared II (NIR-II) fluorescence imaging. Our findings confirm that NIR-IIb fluorescence imaging can be successfully performed using the clinically approved agent ICG. Further clinical application in NIR-IIb region would hopefully be carried out with appropriate ICG-protein solutions.
\end{abstract}

Keywords: fluorescence imaging, NIR-IIb window, indocyanine green, serum, bile, cerebrovascular imaging 


\section{Introduction}

Fluorescence imaging in the near-infrared window (NIR:760-2400 nm) exhibits prominent advantages including real-time imaging, free radiation and superb spatial resolution, compared with traditional imaging techniques such as computed X-ray tomography (CT), positron emission tomography (PET) and magnetic imaging (MRI) ${ }^{[1-5]}$. Among NIR spectral region, imaging performed in the second NIR window (NIRII, 900-1700 nm) outperformed that in the first near-infrared window (NIR-I, 760-900 $\mathrm{nm})$ as the photon scattering effects and endogenous tissue auto-fluorescence signal could be significantly reduced at longer wavelength region ${ }^{[5-8]}$. Moreover, spectral region of 1500-1700 $\mathrm{nm}$ (NIR-IIb) promises even larger tissue penetrating depth and minimum auto-fluorescence due to its longest wavelength in NIR-II window [9] Diversified emitters have been developed for visualizing anatomical structures in recent years ${ }^{[10]}$. However, inorganic nanomaterials including rare-earth doped nanoparticles ${ }^{[11,12]}$ and quantum dots ${ }^{[13]}$ confronted long-term cytotoxicity troubles. Though recently developed organic fluorophores ${ }^{[14-17]}$ exhibited emission beyond $1500 \mathrm{~nm}$, they also encountered low molar extinction coefficients, or long clinical approval process.

Indocyanine green (ICG), approved by the US Food and Drug Administration (FDA), was a conventional NIR-I dye, which had been widely applied for clinic ${ }^{[18]}$. ICG was reported to emit NIR-II fluorescence suitable for noninvasive imaging in blood and lymph vessels in mice ${ }^{[19]}$. Recent clinical applications based on NIR-II fluorescence of ICG have demonstrated the brilliant sensitivity in detecting the primary tumor and extrahepatic metastases ${ }^{[20]}$. Since the inherent defects including poor photostability ${ }^{[6,}$ ${ }^{21]}$ in water, rapid plasma clearance rate in circulation and strong background signal due to its accumulation in liver have impeded ICG's further clinical application, efforts have been tried to stabilize the fluorescent agent. Liposomal formation of ICG and incorporating ICG into PEG have been reported to increase ICG's half-life period in blood ${ }^{[19,22]}$. ICG in fetal bovine serum (FBS) also demonstrated enhanced NIR-II fluorescence intensity ${ }^{[23]}$. So far, ICG fluorescence-guided surgeries are still mainly performed in the NIR-I and NIR-II (specifically in 1000- $1400 \mathrm{~nm}$ ) spectral region.

Here, the fluorescence intensity of ICG dilutions in human serum albumin (HSA), rat bile (containing mucin and plasma proteins) and FBS in the NIR-IIb region were measured. NIR-IIb fluorescence imaging of lymph nodes, extrahepatic biliary tract and cerebrovascular structures with ICG in vivo were also conducted and compared with those in the NIR-II window. The primary objective of the current study was to evaluate the feasibility of performing fluorescence imaging in the NIR-IIb window using a clinically approved agent. 


\section{Materials and Methods}

\subsection{Materials}

Indocyanine green (ICG) was acquired from Sir Run-Run Shaw Hospital, School of Medicine, Zhejiang University. Fetal bovine serum (FBS) and human serum albumin (HSA) were purchased from Dalian Meilun Biotechnology Co., LTD. Phosphatebuffered saline (PBS) was attained from Sinopharm Chemical Reagent Co., LTD., China. Rat Bile in vitro was extracted from live rat ( $\sim 8$ weeks old). Deionized (DI) water was extracted from an Eco-Q15 deionized water system (Shanghai Hitech Instruments Co., Ltd.).

\subsection{Absorption and photoluminescence spectra characterization}

The absorption spectra of ICG dilutions in DI water, 4\% HSA, rat bile and FBS were measured by Shimadzu UV-2550 UV-vis-NIR scanning spectrophotometer. The photoluminescence spectra were recorded by a NIR2000C spectrometer (Everuping Optics Corporation).

\subsection{Optical system for NIR-IIb fluorescence macro-imaging}

The NIR-IIb fluorescence macro-imaging optical system contained excitation part and signal collection light part (Fig.S1). In the excitation part, a $793 \mathrm{~nm}$ laser with collimator was stained on an optical pole. The collimator had an 850 short-pass (850 SP Thorlabs) filter which cut off the longer wavelength of light source. In the signal collection part, an electronic-cooling 2D (640 pixels $\times 512$ pixels) InGaAs camera (TEKWIN SYSTEM, China) which has the good sensitivity at the spectral range from $900 \mathrm{~nm}$ to $1700 \mathrm{~nm}$, was immobilized in an aluminum frame. Below the camera was a prime lens (focal length: $35 \mathrm{~mm}$, Edmund Optics) with antireflection (AR) coating at 800-2000 nm. InGaAs camera thus only collected NIR-IIb (1500-1700 nm) fluorescence signals when equipped with a $1500 \mathrm{~nm}$ LP filter (Thorlabs). The distance between the camera and imaging plane could be adjusted in order to focus.

\subsection{Optical system for NIR-IIb fluorescence mesoscopic/microscopic imaging}

The NIR-IIb fluorescence microscopic imaging optical system (NIRII-MS, Sunnyoptical) adds $900 \mathrm{~nm}$ long-pass dichroic mirror (DMLP) and objectives compared to macroscopic imaging system (Fig.S2). The tube lens had 1000-1700 nm 
anti-reflection. A $793 \mathrm{~nm}$ laser was equipped as excitation source. After $793 \mathrm{~nm}$ laser beams reflect from a $900 \mathrm{~nm}$ long-pass dichroic mirror (DMLP) and pass through an objective, the observed sample is illuminated. The objective could be an air objective lens (LSM03, $\mathrm{WD}=25.1 \mathrm{~mm}$, Thorlabs or LMS05, WD=36 mm, Thorlabs), or an infrared transmission water-immersed object (XLPLN25XWMP2, 25×, NA=1.05, Olympus). NIR-IIb fluorescence images of the sample were recorded with the InGaAs camera after passing through a $1500 \mathrm{~nm}$ long-pass filter (Thorlabs).

\subsection{Ex vivo fluorescence and photostability test}

ICG $(0.1 \mathrm{mg} / \mathrm{mL})$ was dissolved in DI water, 4\% HSA, rat bile and FBS respectively. Four centrifuge tubes which respectively contained $100 \mu \mathrm{L}$ above-mentioned solution, were irradiated by a $793 \mathrm{~nm} \mathrm{CW}$ laser with $60 \mathrm{~mW} / \mathrm{cm}^{2}$ for $200 \mathrm{~min}$ uninterruptedly. The fluorescence images in NIR-IIb region were recorded every 5 min by the InGaAs camera (exposure time $=100 \mathrm{~ms}$ ) under NIR-IIb fluorescence macro-imaging optical system.

\subsection{Sacral/popliteal lymph node NIR-IIb imaging}

Sacral/popliteal lymph node NIR-IIb imaging was also performed by the previous NIR-IIb fluorescence macro-imaging system. ICG $(2 \mathrm{mg} / \mathrm{mL}, 50 \mu \mathrm{L})$ in DI water, $2 \%$ HSA, and 4\% HSA were injected into each digit and the footpad of anesthetic BALB/c nude mice (4 6 weeks female) respectively. Mice were then placed on the imaging platform and taped safely in a position where the Sacral/ popliteal lymph node were observed clearly on InGaAs camera. To attain $1000 \mathrm{~nm}$ LP and $1300 \mathrm{~nm}$ LP macroimage, the filter in front of the camera test surface were changed from $1500 \mathrm{~nm}$ LP to $1000 \mathrm{~nm} \mathrm{LP}$ and $1300 \mathrm{~nm}$ LP accordingly. Imaging conditions: $793 \mathrm{~nm}$ laser power= $22 \mathrm{~mW} / \mathrm{cm}^{2}$ (1000 nm LP), $22 \mathrm{~mW} / \mathrm{cm}^{2}$ (1300 nm LP), and $28 \mathrm{~mW} / \mathrm{cm}^{2}$ (1500 $\mathrm{nm} \mathrm{LP);}$ exposure time $=1 \mathrm{~ms}$ (1000 nm LP), $200 \mathrm{~ms}$ (1300 nm LP), $400 \mathrm{~ms}(1500 \mathrm{~nm} \mathrm{LP})$.

\subsection{In vivo NIR-IIb fluorescence biliary imaging}

In vivo NIR-IIb fluorescence biliary imaging was fulfilled with previous NIR-IIb fluorescence macro-imaging system and NIR-IIb fluorescence mesoscopic imaging optical system. The mouse (6 7 weeks female ICR) was anesthetized via intraperitoneal injection. ICG $(0.2 \mathrm{mg} / \mathrm{mL}, 200 \mu \mathrm{L})$ was injected through caudal vein. Biliary tract was imaged via the optical system after laparotomy was carried out. Imaging conditions: $793 \mathrm{~nm}$ laser power $=70 \mathrm{~mW} / \mathrm{cm}^{2}$ (macroscopy), $190 \mathrm{~mW} / \mathrm{cm}^{2}$ (mesoscopy $\left.2 \mathrm{X}\right), 900$ $\mathrm{mW} / \mathrm{cm}^{2}$ (mesoscopy $4 \mathrm{X}$ ), and $800 \mathrm{~mW} / \mathrm{cm}^{2}$ (mesoscopy $5 \mathrm{X}$ ); exposure time $=100 \mathrm{~ms}$ 
for macroscopy, mesoscopy $2 \mathrm{X}$, mesoscopy $4 \mathrm{X}$ and mesoscopy $5 \mathrm{X}$ imaging.

\subsection{In vivo NIR-IIb fluorescence cerebrovascular imaging}

In vivo NIR-IIb fluorescence cerebrovascular imaging was achieved through NIRIIb fluorescence microscopic imaging optical system. The mice (4 6 weeks female $\mathrm{BALB} / \mathrm{c}$, divided into two groups) were performed microsurgery to remove the skin covering on the skull ${ }^{[24]}$ for the intact skull imaging (group one) or the skull ${ }^{[25]}$ for imaging with a cranial window (group two). The NIR-IIb fluorescence images were recorded immediately after ICG $(2.5 \mathrm{mg} / \mathrm{mL}, 100 \mu \mathrm{L})$ was injected through caudal vein. Imaging conditions for through skull imaging with mesoscopy $5 \mathrm{X}$ objective: $793 \mathrm{~nm}$ laser power $=680 \mathrm{~mW} / \mathrm{cm}^{2}(1000 \mathrm{~nm} \mathrm{LP}), 1.2 \mathrm{~W} / \mathrm{cm}^{2}(1300 \mathrm{~nm} \mathrm{LP})$, and $2.7 \mathrm{~W} / \mathrm{cm}^{2}$ $(1500 \mathrm{~nm} \mathrm{LP})$; exposure time $=7 \mathrm{~ms}$ (1000 nm LP), $300 \mathrm{~ms}(1300 \mathrm{~nm} \mathrm{LP})$, and $900 \mathrm{~ms}$ (1500 nm LP).

\subsection{In vivo PTI damage observation with NIR-IIb fluorescence imaging}

The mice (4 6 weeks female BALB/c) with a cranial window were used for the PTI mouse model. At first, a micro region of the brain was selected and imaged under NIRIIb fluorescence microscopic imaging system. After that, $40 \mu \mathrm{L}$ of photosensitizer Rose Bengal $(10 \mathrm{mg} / \mathrm{mL})$ in PBS was infused via tail vein. Then, the same area on a capillary in the micro region was selected and illuminated for $3 \mathrm{~min}$ with the $532 \mathrm{~nm}$ laser beam (average power after the objective is $60 \mathrm{~mW}$ ) to induce PTI damage. Finally, the micro region with PTI damage was imaged under NIR-IIb fluorescence microscopic imaging system again. Imaging conditions for $25 \mathrm{X}$ NIR-IIb fluorescence microscopic imaging: $793 \mathrm{~nm}$ laser power $=400 \mathrm{~mW}$, exposure time $=100 \mathrm{~ms}$.

\subsection{Ethical approval}

All animal experiments performed in studies were conducted strictly in accordance with the ethical standards of the Institutional Ethical Committee of Animal Experimentation of Zhejiang University. All procedures performed in studies involving human participants were in accordance with the ethical standards of the Clinical Research Ethics Committee of Sir Run-Run Shaw Hospital of Zhejiang University.

\subsection{Data analysis}

Image J software (Version 1.6.0, National Institutes of Health, USA) was applied for quantitative analysis of each fluorescent image. Origin Pro software (Version 9.0, 
OriginLab Company, USA) and Adobe Illustrator CC (Version 2018) was applied for graphs generation.

\section{Results and Discussion}

\subsection{Optical characterization}

The chemical structure of ICG was presented in Fig.1a and its molar mass is 774.96 $\mathrm{g} / \mathrm{mol}$. It was reported that the emission spectrum of ICG in serum could extend to 1300 $\mathrm{nm}{ }^{[23]}$, and the tail emission spectrum of ICG in water could also prolong to NIR-IIb region at in vitro level ${ }^{[19]}$. Nevertheless, NIR-IIb imaging of ICG in vivo hasn't been carried out. We investigated the NIR-IIb fluorescence properties of ICG. The NIR-IIb fluorescence intensity measurement and photostability test were performed. Intensified NIR-IIb fluorescence signal occurred when ICG was dissolved in HSA, rat bile and FBS (Fig.1b). Quantitative analysis demonstrated that ICG in rat bile emitted 6.5-fold higher NIR-IIb fluorescence intensity than ICG in DI water. Fluorescence intensity of ICG in both FBS solution (as purchased) and 4\% HSA solution (concentration $=40$ $\mathrm{mg} / \mathrm{mL}$ ) increased to $\sim 5.2$-fold compared with that in DI water (Fig.1c). Although FBS and HSA have been reported to enhance fluorescence intensity of other small-molecular NIR-II probes ${ }^{[21,26,27]}$, the intensification of NIR-IIb fluorescence of ICG was firstly demonstrated.

ICG showed red-shifted and enhanced absorption spectra when dissolved in 4\% HSA, rat bile and FBS (Fig.1d). The absorption peaked at $803.5 \mathrm{~nm}$ (in FBS solution), 803.0 $\mathrm{nm}$ (in rat bile solution) and $800.5 \mathrm{~nm}$ (in 4\% HSA solution), red-shifting about $20 \mathrm{~nm}$ compared with the peak in DI water $(778.5 \mathrm{~nm})$. Peak absorption cross-sections of ICG in 4\% HSA, rat bile and FBS increased up to $~ 2.0$-fold compared with that in DI water. As a result, the photoluminescence spectra of ICG in NIR-II window were also intensified under the $793 \mathrm{~nm}$ excitation, when dissolved in 4\% HSA, rat bile and FBS (Fig.1e). The inserted spectra further confirmed the more bright fluorescence of ICG in 4\% HSA, rat bile and FBS. In addition, under the continuous $793 \mathrm{~nm}$ laser excitation, the NIR-IIb fluorescence decay times of ICG in 4\% HSA (70 min), rat bile (more than $200 \mathrm{~min}$ ) and FBS (158 min) was longer than that in DI water (63 min) (Fig.S3). Combining the aforementioned results, we know that ICG in 4\% HSA, rat bile and FBS emitted much higher and more lasting NIR-IIb fluorescence intensity than ICG in DI water, which are suitable for the following NIR-IIb fluorescence functional bioimaging.

\subsection{NIR-IIb fluorescence imaging for lymph system}


ICG has been applied for the resection of sentinel lymph nodes (SLN) during fluorescence-guided surgeries ${ }^{[28,29]}$. However, the deficiencies including poor contrast and low resolution in the NIR-I window were evident ${ }^{[21,30]}$. Organic small molecules such as $\mathrm{CH} 1055^{[30]}$ and $\mathrm{CH}-4 \mathrm{~T}^{[21]}$ were developed in succession and allowed high SBR and resolution in NIR-II fluorescence imaging of lymph. Though it was reported NIR-II imaging of lymph vessels with ICG in water preserved contrast up to about 1400 $\mathrm{nm},{ }^{[19]}$ imaging of lymph system with ICG in NIR-IIb region (1500- $1700 \mathrm{~nm}$ ) has not been demonstrated.

We studied the effectiveness of NIR-IIb fluorescence imaging with ICG in lymphatic drainage in vivo. ICG mixed with $\mathrm{HSA}(2 \mathrm{mg} / \mathrm{mL}, 50 \mu \mathrm{L})$ was injected intradermally at the rear paw of nude mice. It was evident that NIR-II $(1000 \mathrm{~nm}, 1300 \mathrm{~nm}$ and $1500 \mathrm{~nm}$ long pass filter) fluorescence imaging for both lymph vessels and lymph nodes (LNs) exhibited enhanced intensity as the proportion of HSA increased from $0 \%$ to $4 \%$ (Fig.2a). Quantitative analysis of the NIR-IIb fluorescence of sciatic LNs stained with ICG in 4\% HSA showed 11.3 (left) to 5.47 (right) fold increase in intensity, compared with those stained with ICG in 0\% HSA (Fig.2b). Moreover, the results implied that HSA promoted ICG to flow from afferent lymph vessels to popliteal LN and efferent lymphatic vessels to sciatic LN. Thus, fluorescence imaging using ICG in 4\% HSA (HSA 4\% row in Fig.2a) was applied for further analysis. The lymphatic drainage of the same mouse was imaged under the $1000 \mathrm{~nm} \mathrm{LP,} 1300 \mathrm{~nm}$ LP and $1500 \mathrm{~nm}$ LP respectively. The full width of half-maximum (FWHM) of sciatic LN beyond $1500 \mathrm{~nm}$ was measured as $0.842 \mathrm{~mm}$, lower than those beyond $1300 \mathrm{~nm}(1.185 \mathrm{~mm})$ and 1000 $\mathrm{nm}(1.346 \mathrm{~mm}$ ) (Fig.2c). The SBR of the same LN beyond $1500 \mathrm{~nm}$ was calculated as 24.1, much higher than those beyond $1300 \mathrm{~nm}$ (15.9) and $1000 \mathrm{~nm}$ (7.30). Similarly, the imaging of afferent lymphatic vessels beyond $1500 \mathrm{~nm}$ presented highest contrast $(\mathrm{SBR}=3.40)$ and resolution $(\mathrm{FWHM}=0.530 \mathrm{~mm})$ compared with those beyond 1300 $\mathrm{nm}(\mathrm{SBR}=2.35, \mathrm{FWHM}=1.057 \mathrm{~mm})$ and $1000 \mathrm{~nm}(\mathrm{SBR}=1.32, \mathrm{FWHM}=0.970 \mathrm{~mm})$ (Fig.2d).

Therefore, NIR-IIb fluorescence imaging of lymph drainage system assisted with ICG in HSA solution demonstrated strong potential for future surgical application.

\subsection{NIR-IIb imaging for biliary tract}

Traditional laparoscopic cholecystectomy (LC) have encountered many problems such as risks of biliary anatomical variations and bile duct injuries ${ }^{[31-33]}$. NIR-I fluorescence imaging with ICG was reported in detecting bile excretion during hepatoportoenterostomy for the treatments of biliary atresia (BA) ${ }^{[34]}$. A systematic review illustrated that extrahepatic bile duct visualization assisted with ICG NIR-I fluorescence system was superior to intraoperative cholangiogram ${ }^{[35]}$. However, it was 
mentioned that high fluorescence signal of liver parenchyma have impeded the contrast of bile duct ${ }^{[18]}$, making it difficult to assess the bile leakage ${ }^{[36,37]}$. Therefore, it is critically important to develop an imaging technology to visualize the biliary tract in high contrast, especially for the structures adjacent to the liver tissue.

Our previous study has confirmed the extrahepatic cholangiography in NIR-II window outperformed the conventional NIR-I imaging ${ }^{[38]}$. Herein, we further investigated the capability of ICG for NIR-IIb fluorescence imaging of biliary tract at both macroscopic and mesoscopic level (Fig.3a-d). Macroscopic imaging conditions which we applied were mild: ICG dosage was $0.2 \mathrm{mg} / \mathrm{mL}, 200 \mu \mathrm{L} ; 793 \mathrm{~nm}$ laser excitation power was $70 \mathrm{~mW} / \mathrm{cm}^{2}$; and exposure time of InGaAs camera was $100 \mathrm{~ms}$. Macroscopic imaging showed that the common bile duct (CBD) was bright with high contrast compared with the liver parenchyma and duodenum (Fig.3a). Though ICG was accumulated in liver parenchyma, the fluorescence intensity of liver in NIR-IIb window was lower than that in biliary tract. We speculated that the large amount of water in liver absorbed and weakened its NIR-IIb fluorescence intensity. Additionally, cystic duct was clearly visible (FWHM=0.299 $\mathrm{mm}$ and $\mathrm{SBR}=7.56$, Fig.3e). In order to precisely visualize the structure of biliary tract, three kinds of objectives with different magnifications were employed for mesoscopic imaging. The sharpness of cystic duct was apparent in Fig.3b-d. FWHM of cystic duct improved compared with macroscopic imaging. Moreover, we were able to visualize the ICG flux in the biliary tract at a speed of 25 frames per second, even there existed the interference from the rapid heart-beat (Movie S1).

Consequently, it would be available to detect the potential bile leakage or other biliary lesions more accurately benefiting from the bright fluorescence signal in the biliary tract and the weakened background signal from the liver tissue in the NIR-IIb window.

\subsection{NIR-IIb fluorescence wide-field microscopic cerebrovascular imaging}

2-photon fluorescence cerebrovascular imaging (exciting ICG with femtosecond pulses at $1617 \mathrm{~nm}$ ) realized $2000 \mu \mathrm{m}$ imaging depth ${ }^{[39]}$. Moreover, microscopic cerebrovascular imaging assisted with ICG in the NIR-II region has been performed with large imaging depth $(850 \mu \mathrm{m})$ and high resolution $(10.71 \mu \mathrm{m})$ in mice with a cranial window ${ }^{[23]}$. We then evaluated the feasibility of NIR-IIb fluorescence widefield microscopic cerebrovascular imaging with ICG. ICG $(2.5 \mathrm{mg} / \mathrm{mL}, 100 \mu \mathrm{L})$ was intravenously injected into mice with intact skull. NIR-IIb image with a large field (Fig.4c) displayed clearer blood vessels compared with NIR-II image (Fig.4a-b). This could be possibly explained that intact skull increased the scattering of shortwavelength NIR-II fluorescence. Gaussian fitting analysis of top right vessel showed 
that NIR-IIb fluorescence imaging has smallest FWHM and highest SBR, compared with images performed with $1000 \mathrm{~nm}$ LP and 13000 nm LP (Fig.4d). Furthermore, a small blood vessel at medium position displayed $7.87 \mu \mathrm{m}$ spatial resolution, while vessel structures in $1000 \mathrm{~nm}$ LP and $1300 \mathrm{~nm}$ LP groups could hardly be resolved through background noise and became quite indistinguishable (Fig.4e).

Considering the high photon scattering of skull, mice with a cranial window were also imaged. $400 \mu \mathrm{m}$ imaging depth and capillary vessels as small as $3.75 \mu \mathrm{m}$ were displayed (Fig.5a). Then, photo thrombotic ischemia (PTI) induction was carried out. A $532 \mathrm{~nm}$ laser beam was employed to excite photosensitizer (rose Bengal) for PTI induction. Released singlet oxygen through photosensitizer would adhere to the endothelial cells of blood vessels and damage them, causing cerebral thrombosis ${ }^{[40]}$. Cerebral vessels were imaged before and after PTI formation (Fig.5b). Dashed lines manifested the corrupted vessels. Abnormal increases of fluorescence intensity in the vessels displayed the obstructed blood which was highlighted using solid line. Therefore, our study suggested the possibility of diagnosis of brain diseases via NIR$\mathrm{IIb}$ fluorescence microscopic imaging with ICG.

\section{Conclusion}

In summary, we demonstrated the enhanced NIR-IIb fluorescence intensity of ICG when dissolved in the 4\% HSA, rat bile and FBS solution. Benefiting from the reduced auto-fluorescence in the NIR-IIb region, the lymphatic drainage and the biliary tract could be imaged with higher contrast and resolution. Moreover, NIR-IIb fluorescence microscopic imaging assisted with ICG was firstly presented. Therefore, ICG bears great potential for translation of NIR-IIb imaging technology to clinical trial with the help of proteins. Fluorescence imaging in the NIR-IIb window could also be successfully performed using the clinical approved agent ICG and might be a promising technology for clinical diagnosis.

\section{Acknowledgments}

This work was jointly supported by the National Natural Science Foundation of China (61735016 and 61975172), and the Zhejiang Provincial Natural Science Foundation of China (LR17F050001).

\section{Conflict of interest}


The authors declare that they have no conflict of interest.

\section{Author contributions}

Mubin $\mathrm{He}$ was mainly responsible for the design and operation of imaging experiments and data analysis. Di Wu mainly assisted with biliary imaging experiment, provided comments and revised the manuscript. Yuhuang Zhang assisted with data analysis and characterization experiment. Xiaoxiao Fan mainly assisted with lymph imaging experiment. Jun Qian was the corresponding author of this article, designed the imaging systems and provided guidance in the entire research process.

\section{Appendix A. Supplementary materials}

Supplementary materials to this article can be found online.

\section{References}

[1] Kobayashi H, Choyke PL. Target-cancer-cell-specific activatable fluorescence imaging probes: Rational design and in vivo applications. Accounts of chemical research, 2011, 44: 83-90

[2] Owens EA, Henary M, El Fakhri G, et al. Tissue-specific near-infrared fluorescence imaging. Accounts of chemical research, 2016, 49: 1731-1740

[3] Hong G, Antaris AL, Dai H. Near-infrared fluorophores for biomedical imaging. Nature biomedical engineering, 2017, 1:

[4] Miao Q, Xie C, Zhen X, et al. Molecular afterglow imaging with bright, biodegradable polymer nanoparticles. Nature biotechnology, 2017, 35: 1102-1110

[5] Kenry, Duan Y, Liu B. Recent advances of optical imaging in the second nearinfrared window. Advanced materials, 2018, 30: 1802394

[6] Shou K, Qu C, Sun Y, et al. Multifunctional biomedical imaging in physiological and pathological conditions using a nir-ii probe. Advanced functional materials, 2017, 27: 1700995

[7] Qi J, Sun C, Zebibula A, et al. Real-time and high-resolution bioimaging with bright aggregation-induced emission dots in short-wave infrared region. Advanced materials, 2018, 30: e1706856

[8] Tang Y, Li Y, Hu X, et al. "Dual lock-and-key" controlled nanoprobes for ultrahigh 
specific fluorescence imaging in the second near-infrared window. Advanced materials, 2018, 30:

[9] Diao S, Blackburn J, Hong G, et al. Fluorescence imaging in vivo at wavelengths beyond $1500 \mathrm{~nm}$. Angewandte Chemie (International ed in English), 2015, 54:

[10]Zhang MX, Yue JY, Cui R, et al. Bright quantum dots emitting at similar to 1,600 $\mathrm{nm}$ in the nir-iib window for deep tissue fluorescence imaging. P Natl Acad Sci USA, 2018, 115: 6590-6595

[11] Naczynski D, Tan M, Zevon M, et al. Rare-earth-doped biological composites as in vivo shortwave infrared reporters. Nature communications, 2013, 4: 2199

[12] Wang R, Li X, Zhou L, et al. Epitaxial seeded growth of rare-earth nanocrystals with efficient $800 \mathrm{~nm}$ near-infrared to $1525 \mathrm{~nm}$ short-wavelength infrared downconversion photoluminescence for in vivo bioimaging. Angew Chem Int Ed, 2014, 126: $12282-12286$

[13]Bruns O, Bischof T, Harris D, et al. Next-generation in vivo optical imaging with short-wave infrared quantum dots. Nature biomedical engineering, 2017, 1: 0056

[14] Sun C, Li B, Zhao M, et al. J-aggregates of cyanine dye for nir-ii in vivo dynamic vascular imaging beyond $1500 \mathrm{~nm}$. Journal of the American Chemical Society, 2019, 141: 19221-19225

[15]Li Y, Cai Z, Liu S, et al. Design of aiegens for near-infrared iib imaging through structural modulation at molecular and morphological levels. Nat Commun, 2020, 11 : 1255

[16]Li Y, Liu Y, Li Q, et al. Novel nir-ii organic fluorophores for bioimaging beyond $1550 \mathrm{~nm}$. Chemical Science, 2020, 11: 2621-2626

[17]Li Q, Ding Q, Li Y, et al. Novel small-molecule fluorophores for in vivo nir-iia and nir-iib imaging. Chem Commun (Camb), 2020, 56: 3289-3292

[18]Baiocchi GL, Diana M, Boni L. Indocyanine green-based fluorescence imaging in visceral and hepatobiliary and pancreatic surgery: State of the art and future directions. World journal of gastroenterology, 2018, 24: 2921-2930

[19]Carr JA, Franke D, Caram JR, et al. Shortwave infrared fluorescence imaging with the clinically approved near-infrared dye indocyanine green. P Natl Acad Sci USA, 2018, 115: 4465-4470

[20]Hu Z, Fang C, Li B, et al. First-in-human liver-tumour surgery guided by multispectral fluorescence imaging in the visible and near-infrared-i/ii windows. Nature biomedical engineering, 2019,

[21] Antaris AL, Chen H, Diao S, et al. A high quantum yield molecule-protein complex fluorophore for near-infrared ii imaging. Nat Commun, 2017, 8: 15269

[22]Bhavane R, Starosolski Z, Stupin I, et al. Nir-ii fluorescence imaging using indocyanine green nanoparticles. Scientific reports, 2018, 8: 14455

[23] Yu X, Feng Z, Cai Z, et al. Deciphering of cerebrovasculatures via icg-assisted nir- 
ii fluorescence microscopy. Journal of Materials Chemistry B, 2019, [24]Zhang C, Feng W, Zhao Y, et al. A large, switchable optical clearing skull window for cerebrovascular imaging. Theranostics, 2018, 8: 2696-2708

[25]Qian J, Wang D, Cai FH, et al. Observation of multiphoton-induced fluorescence from graphene oxide nanoparticles and applications in in vivo functional bioimaging. Angew Chem Int Ed Engl, 2012, 51: 10570-10575

[26]Li D, Qu C, Liu Q, et al. Monitoring the real - time circulatory system - related physiological and pathological processes in vivo using a multifunctional nir - ii probe. Advanced Functional Materials, 2019, 1906343

[27]Feng Z, Yu X, Jiang M, et al. Excretable ir-820 for in vivo nir-ii fluorescence cerebrovascular imaging and photothermal therapy of subcutaneous tumor. Theranostics, 2019, 9: 5706-5719

[28] Troyan SL, Kianzad V, Gibbs-Strauss SL, et al. The flare intraoperative nearinfrared fluorescence imaging system: A first-in-human clinical trial in breast cancer sentinel lymph node mapping. Annals of surgical oncology, 2009, 16: 2943-2952

[29]Chi C, Ye J, Ding H, et al. Use of indocyanine green for detecting the sentinel lymph node in breast cancer patients: From preclinical evaluation to clinical validation. PLoS One, 2013, 8: e83927-e83927

[30] Antaris AL, Chen H, Cheng K, et al. A small-molecule dye for nir-ii imaging. Nat Mater, 2016, 15: 235-242

[31]Perissat J. Laparoscopic cholecystectomy: The european experience. The American Journal of Surgery, 1993, 165: 444-449

[32]Flum DR, Dellinger EP, Cheadle A, et al. Intraoperative cholangiography and risk of common bile duct injury during cholecystectomy. JAMA, 2003, 289: 1639-1644 [33] Giger U, Ouaissi M, Hsu Schmitz S-F, et al. Bile duct injury and use of cholangiography during laparoscopic cholecystectomy. The British journal of surgery, 2011, 98: 391-396

[34]Hirayama Y, Iinuma Y, Yokoyama N, et al. Near-infrared fluorescence cholangiography with indocyanine green for biliary atresia. Real-time imaging during the kasai procedure: A pilot study. Pediatric surgery international, 2015, 31: 1177-1182 [35] Vlek SL, van Dam DA, Rubinstein SM, et al. Biliary tract visualization using nearinfrared imaging with indocyanine green during laparoscopic cholecystectomy: Results of a systematic review. Surgical endoscopy, 2017, 31: 2731-2742

[36] Kaibori M, Ishizaki M, Matsui $\mathrm{K}$, et al. Intraoperative indocyanine green fluorescent imaging for prevention of bile leakage after hepatic resection. Surgery, 2011, 150: $91-98$

[37] Sakaguchi T, Suzuki A, Unno N, et al. Bile leak test by indocyanine green fluorescence images after hepatectomy. American journal of surgery, 2010, 200: e19- 
[38]Wu D, Xue D, Zhou J, et al. Extrahepatic cholangiography in near-infrared ii window with the clinically approved fluorescence agent indocyanine green: A promising imaging technology for intraoperative diagnosis. Theranostics, 2020, 10: 3636-3651

[39]Cheng H, Tong S, Deng X, et al. Deep-brain 2-photon fluorescence microscopy in vivo excited at the $1700 \mathrm{~nm}$ window. Optics letters, 2019, 44: 4432-4435

[40] Wu W, Mao D, Hu F, et al. A highly efficient and photostable photosensitizer with near-infrared aggregation-induced emission for image-guided photodynamic anticancer therapy. Advanced materials, 2017, 29: 1700548

Figures:

(a)

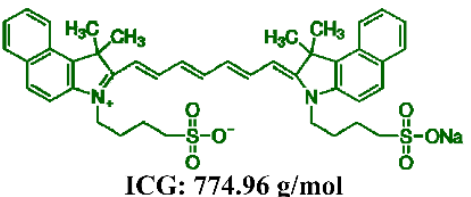

(b)

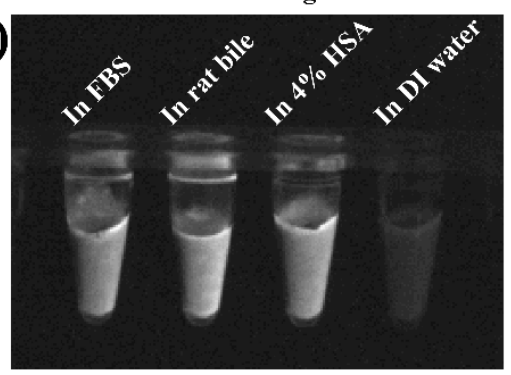

(c)

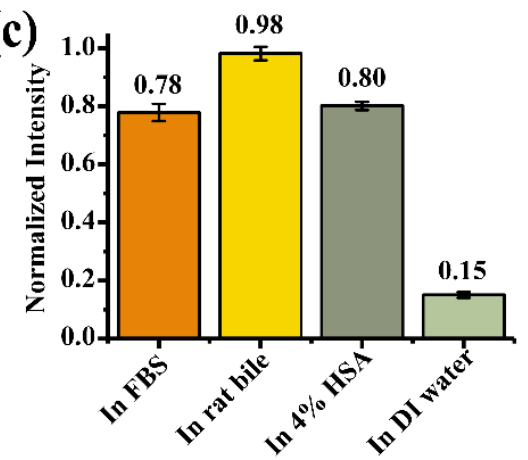

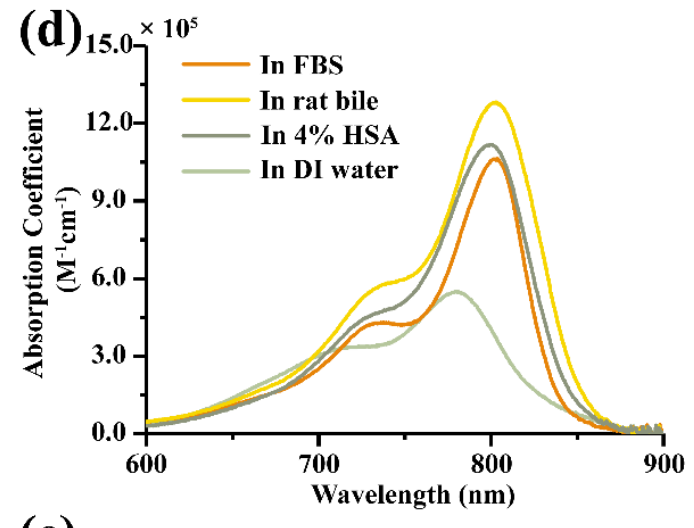

(e)

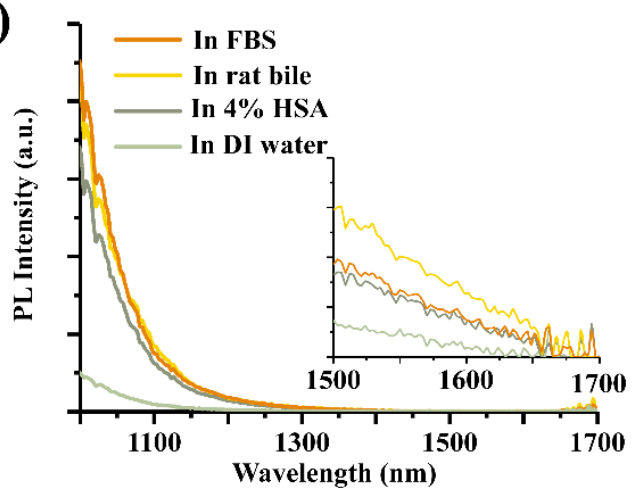

Fig. 1. Optical characterizations of indocyanine green (ICG). (a) Molecular structure of ICG. (b) The NIR-IIb fluorescence signals of ICG $(0.1 \mathrm{mg} / \mathrm{mL})$ in FBS solution, rat bile, 4\% HSA solution and DI water, under $793 \mathrm{~nm}$ laser excitation $\left(60 \mathrm{~mW} / \mathrm{cm}^{2}\right) .1500$ $\mathrm{nm}$ long-pass filter was adopted and the exposure time was $100 \mathrm{~ms}$. (c) The calculated NIR-IIb intensities of ICG in various protein solutions, according to the images of Fig. 1B. (d) The absorption coefficient of ICG in FBS solution, rat bile, 4\% HSA solution and DI water. (e) The photoluminescence spectra of ICG $(0.1 \mathrm{mg} / \mathrm{mL})$ in NIR-II region; insert: spectra of ICG in NIR-IIb region. ICG $(0.1 \mathrm{mg} / \mathrm{mL})$ in DI water, $4 \% \mathrm{HSA}$, rat bile and FBS were excited under the same condition (793 nm laser). 

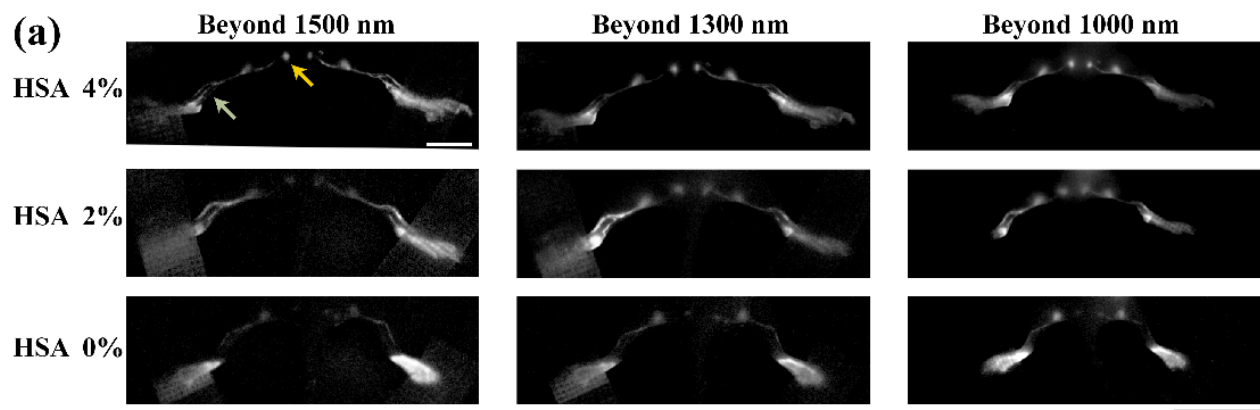

(b)

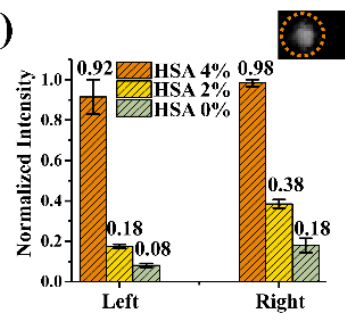

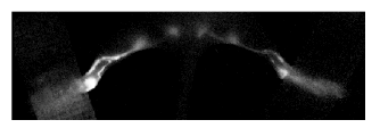

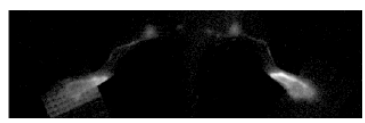

(c) Beyond $1500 \mathrm{~nm}$

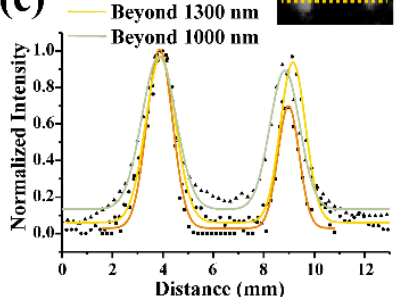

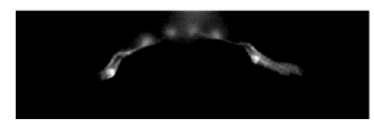

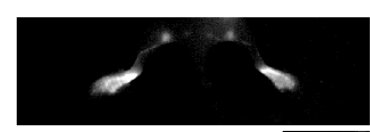

(d)

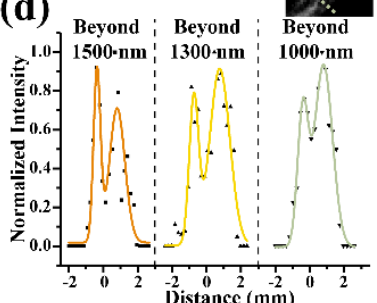

Fig. 2. In vivo NIR-II fluorescence imaging of lymphatic drainage. (a) Fluorescence images of lymphatic drainage in various spectral regions. The parameters (in supplementary method) were adapted to obtain optimal image contrast. Scale bar $=10$ mm. (b) NIR-IIb fluorescence intensities of sciatic lymph nodes of the mouse, which was injected with ICG in HSA solution (the proportion of HSA increased from $0 \%$ to $4 \%$ ). (c) Gaussian fitting curves of the NIR-IIb fluorescence intensities of sciatic lymph nodes. The left sciatic lymph node's FWHM=0.842 mm (1500 nm LP), $1.185 \mathrm{~mm}$ (1300 nm LP), 1.346 mm (1000 nm LP); SBR=24.1 (1500 nm LP), 15.9 (1300 nm LP), 7.30 (1000 nm LP). (d) Gaussian fitting curves of the NIR-IIb fluorescence intensities of efferent lymph vessels in left hindlimb. The left efferent lymph vessel's FWHM= $0.530 \mathrm{~mm}$ (1500 nm LP), $1.057 \mathrm{~mm}$ (1300 nm LP), $0.970 \mathrm{~mm}$ (1000 nm LP); SBR= 3.40 (1500 nm LP), 2.35 (1300 nm LP), 1.32 (1000 nm LP). 


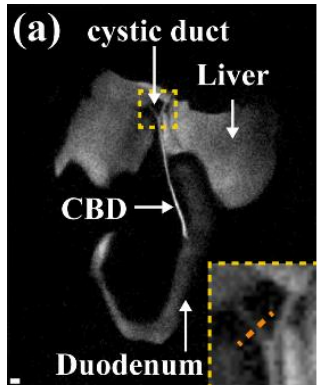

(e)

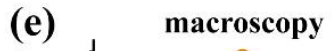

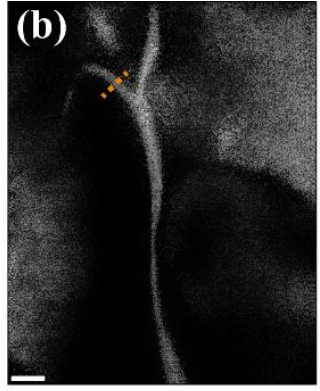

mesoscopy $2 \mathrm{X}$

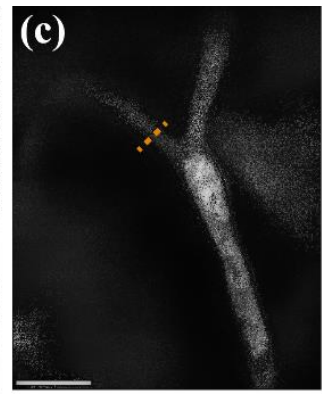

mesoscopy $4 \mathrm{X}$

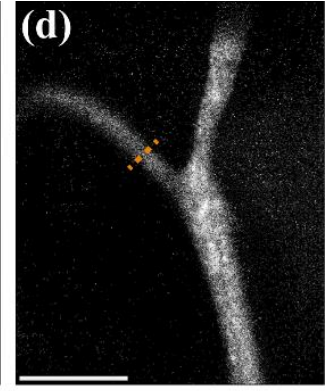

mesoscopy $5 X$

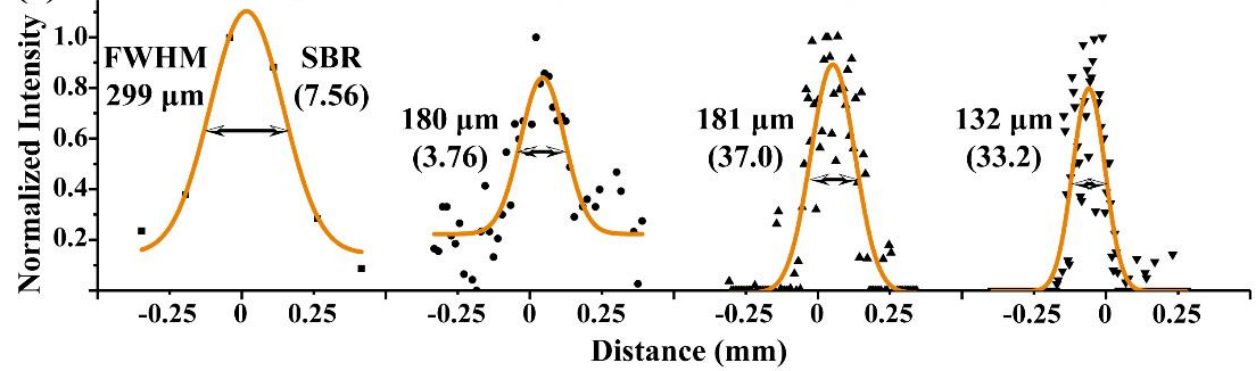

Fig. 3. Real-time NIR-IIb fluorescence (a) macroscopic imaging and (b-d) mesoscopic imaging of biliary tract in the mouse intravenously injected with ICG $(0.2 \mathrm{mg} / \mathrm{mL}, 200$ $\mu \mathrm{L})$. The magnifications of the objective were $2 \mathrm{X}, 4 \mathrm{X}$, and $5 \mathrm{X}$ respectively. Scale bar= $1000 \mu \mathrm{m}$. (e) Gaussian fitting curves of cystic duct pointed in a-d. CBD: common bile duct.
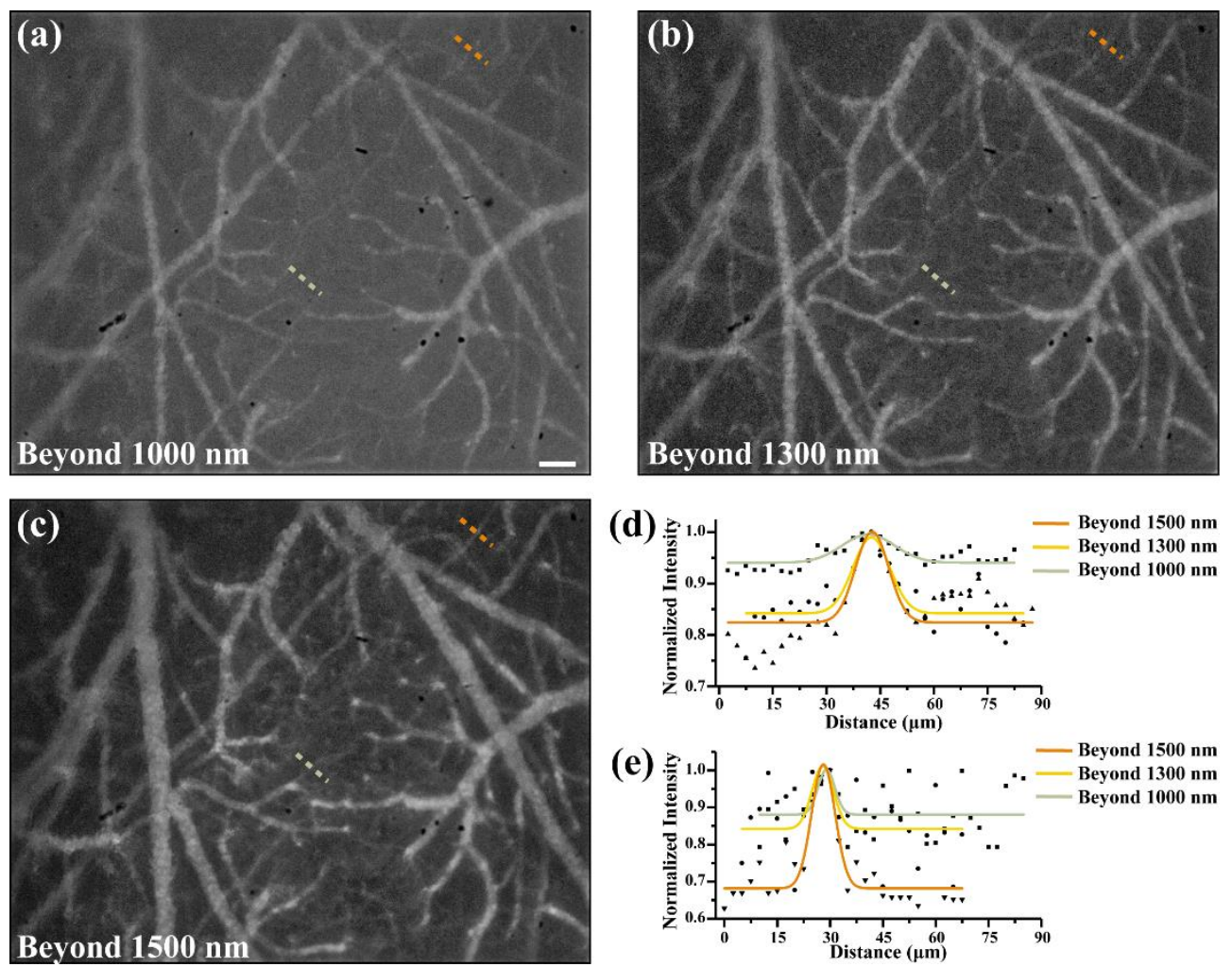

Fig. 4: Real-time NIR-IIb fluorescence microscopic cerebrovascular imaging of the mouse with intact skull treated with ICG: (a-c) Images of brain blood vessels with 1000 
$\mathrm{nm}, 1300 \mathrm{~nm}$ and $1500 \mathrm{~nm}$ long-pass filters respectively. The mouse was intravenously injected with ICG $(2.5 \mathrm{mg} / \mathrm{mL}, 100 \mu \mathrm{L})$, excited by a $793 \mathrm{~nm}$ laser. Images were collected via an objective with the magnification of $5 \mathrm{X}$. Scale bar $=100 \mu \mathrm{m}$. (d) Gaussian fitting curves of blood vessels in a-c at top right positions. FWHM=10.90 $\mu \mathrm{m}$ (1500 nm LP), $12.11 \mu \mathrm{m}$ (1300 nm LP), $17.44 \mu \mathrm{m}$ (1000 nm LP); SBR= 1.21 (1500 nm LP), 1.17 (1300 nm LP), and 1.05 (1000 nm LP). (e) Gaussian fitting curves of blood vessels in a-c at medium positions; FWHM=7.87 $\mu \mathrm{m}$ (1500 nm LP), $7.17 \mu \mathrm{m}$ $(1300 \mathrm{~nm} \mathrm{LP})$, and $5.61 \mu \mathrm{m}$ (1000 nm LP); SBR= 1.49 (1500 nm LP), 1.20 (1300 nm $\mathrm{LP}$ ), and 1.13 (1000 nm LP); Gaussian fitting $\mathrm{R}^{2}=0.830$ (1500 nm LP), 0.230 (1300 nm LP), and 0.149 (1000 nm LP).

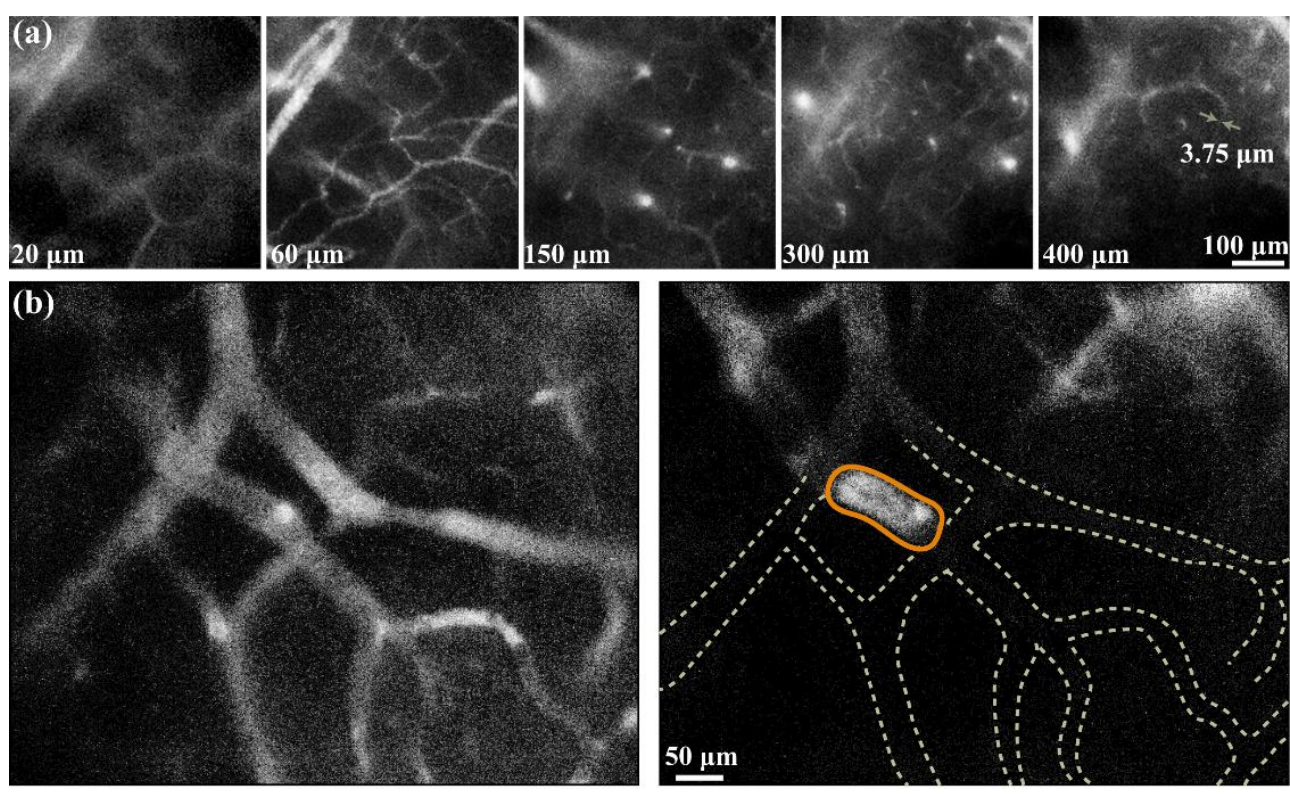

Fig. 5. Real-time NIR-IIb fluorescence microscopic cerebrovascular imaging of the mouse with a cranial window intravenously injected with ICG $(2.5 \mathrm{mg} / \mathrm{mL}, 100 \mu \mathrm{L})$ : (a) Cerebral vasculatures images at various depths $(20-400 \mu \mathrm{m})$ recorded by $25 \mathrm{X}$ objective (XLPLN25XWMP2, NA = 1.05, Olympus). (b) Cerebral vasculatures images before and after the formation of photo thrombotic ischemia (PTI). 